\title{
Statischer Stiffness-Index von zwei Multikomponenten-Bandagen- Kompressionssystemen: Ergebnisse einer randomisierten kontrollierten Studie an gesunden Probanden
}

\section{Static Stiffness Index of Two Multicomponent Bandage Compression Systems: Results of a Randomized Controlled Trial on Healthy Volunteers}

\section{(c) (P)}

\author{
Autoren \\ Jean-Patrick Benigni ${ }^{1}$, Florence Balet ${ }^{2}$, Bérengère Guidet ${ }^{3}$, Olivier Tacca ${ }^{4}$
}

Institute

1 Angiologe, Saint Mandé, Frankreich

2 Freiberufliche Krankenschwester, Pélissane, Frankreich

3 Klinische Projektleiterin, Intertek Clinical Research Services, Paris, Frankreich

4 Direktor der Abteilung für präklinische und klinische Forschung, Urgo Research Innovation and Development, Chenôve, Frankreich

\section{Schlüsselwörter}

Mehrkomponentenkompressionssystem, Statischer

Steifigkeitsindex, Grenzflächendruck

\section{Key words}

multicomponent compression system, static stiffness index, interface pressure

online publiziert 21.12 .2021

Bibliografie

Phlebologie 2022; 51: 21-30

DOI $10.1055 / a-1487-4194$

ISSN 0939-978X

(C) 2021. The Author(s).

This is an open access article published by Thieme under the terms of the Creative Commons Attribution License, permitting unrestricted use,

distribution, and reproduction so long as the original work is properly cited. (https://creativecommons.org/licenses/by/4.0/)

Georg Thieme Verlag KG, Rüdigerstraße 14,

70469 Stuttgart, Germany

Korrespondenzadresse

Jean-Patrick Benigni, MD

15 avenue du Général de Gaulle, 94160 Saint Mandé,

Frankreich

benigni.jp@orange.fr

\section{ZUSAMMENFASSUNG}

Zweck Vergleich der Leistung von zwei MehrkomponentenKompressionssystemen.

Methoden In dieser randomisierten, kontrollierten Studie wurden beide Beine von 25 gesunden Probanden nach dem
Zufallsprinzip entweder mit einem Kompressionssystem der neuen Generation (UrgoK1, eine einzige Binde) oder einem etablierten System (UrgoK2, zwei Binden) als Kontrolle bandagiert. Beide Systeme wurden Tag und Nacht getragen. Arbeits- und Ruhegrenzflächendruck wurden unmittelbar nach dem Anlegen und nach $4 \mathrm{~h}, 24 \mathrm{~h}, 48 \mathrm{~h}$ und $72 \mathrm{~h}$ gemessen und der Static Stiffness Index (SSI) berechnet.

Ergebnisse Nach 4 Stunden wurden mit beiden Systemen ähnlich hohe Arbeitsdrücke und mäßige Ruhedrücke registriert. Im Zeitverlauf folgten die Druckänderungen und des SSI den gleichen Kurven. Nach 48 h wurde ein SSI $\geq 10 \mathrm{mmHg}$ bei $88 \%$ der getesteten und $76 \%$ der Kontrollsysteme erreicht, was die Nichtunterlegenheit des Testsystems bestätigt $(p=0,016)$. Beide Systeme wiesen gute Halteeigenschaften auf und waren gut verträglich, aber das getestete System wurde von der Mehrheit der Probanden als deutlich angenehmer empfunden und schließlich dem Kontrollsystem vorgezogen.

Schlussfolgerung Das neue Kompressionssystem erreichte ähnliche Leistungen wie die Kontrolle, aber seine bessere Akzeptanz könnte ein Vorteil für die Patientencompliance sein. Diese vielversprechenden Ergebnisse müssen in einer klinischen Studie an Patienten mit Unterschenkelulcera und/ oder Ödemen bestätigt werden.

\section{ABSTRACT}

Purpose To compare the performances of two multicomponent compression systems.

Methods In this randomised controlled trial, both legs of 25 healthy volunteers were randomly bandaged with either a new generation of compression system (UrgoK1, one unique bandage) or an established system (UrgoK2, two bandages), as a control. Both systems were worn day and night. Working and resting interfaces pressures were measured, and Static Stiffness Index (SSI) calculated, immediately after application and after $4 \mathrm{~h}, 24 \mathrm{~h}, 48 \mathrm{~h}$ and $72 \mathrm{~h}$.

Results After 4 hours, similar high working pressures and moderate resting pressures were registered with both systems. Over time, changes in pressures and in SSI followed 
the same curves. After $48 \mathrm{~h}$, a SSI $\geq 10 \mathrm{mmHg}$ was reached with $88 \%$ and $76 \%$ of the tested and control systems, respectively, validating the non-inferior rigidity of the tested system $(p=0.016)$. Both systems presented good holding properties and were well tolerated, but the tested system was perceived as significantly more comfortable and eventually preferred to the control system by the majority of the volunteers.
Conclusion The new compression system achieved similar performances to the control, but its better acceptability could become an asset for patients' compliance. These promising results need to be confirmed in a clinical study on patients with leg ulcers and/or oedema.

\section{Einführung}

Die Kompressionstherapie ist nach wie vor der Eckpfeiler aller therapeutischen Ansätze bei der Behandlung der chronisch venösen Insuffizienz (CVI). Diese ist eine venöse Durchblutungsstörung, die durch venöse Hypertonie, venöse Stase, Ödeme und im Endstadium durch venöse Beinulzera, deren Wundheilung mehrere Monate dauert und die eine hohe Rezidivrate aufweisen [1-3], gekennzeichnet ist.

Um den hydrostatischen Kräften der venösen Hypertonie entgegenzuwirken und den gestörten venösen Fluss wiederherzustellen, muss kontinuierlich ein gewisser Druck von außen auf das Bein der zu behandelnden Person ausgeübt werden. In Arbeitsstellung (wenn sie sitzt, steht oder geht) ist ein hoher Druck von mindestens 30-50 mmHg erforderlich, um die Beinvenen zu verengen, während in Rückenlage ein niedrigerer Druck von über $15-25 \mathrm{mmHg}$ ausreicht, um tief liegende Venen zu verengen und den venösen Fluss zu beschleunigen [4]. Die Kompression mit einem unelastischen Material erhöht auch die Massagewirkung der Muskelpumpe bei ambulant Behandelten und verbessert so den venösen Rückfluss. Eine hohe Venenkompression stellt nachweislich die Venenklappenfunktion wieder her, reduziert den venösen Reflux und verbessert den venösen Rückfluss, lindert Schmerzen, reduziert Ödeme und fördert den Wundverschluss von durch CVI verursachten Beinulzera [4-6].

Um diesen Druck auszuüben, stehen den medizinischen Fachkräften verschiedene medizinische Hilfsmittel zur Verfügung: Kurzzugbinden, Langzugbinden, Mehrkomponentensysteme, Kompressionsstrumpf-Sets oder verstellbare Kompressionsverbände. Auf der Grundlage von Metaanalysen und systematischen Übersichten der klinischen Evidenz werden in den aktuellen Leitlinien Mehrkomponentensysteme für die Erstbehandlung von venösen Beinulzera empfohlen, da sie eine höhere Verschlussrate und schnellere Heilung ermöglichen [1, 2, 6-10].

Theoretisch sollen alle diese Mehrkomponentensysteme einen Arbeitsdruck liefern, der hoch genug ist, um der Schwerkraft in aufrechter Position entgegenzuwirken, aber nicht so hoch, dass er Schäden oder Schmerzen verursachen könnte, sowie einen erträglichen Ruhedruck, sodass das System Tag und Nacht bequem getragen werden kann. Die Steifigkeit dieser Systeme, die durch einen statischen Steifigkeitsindex von $\geq 10 \mathrm{mmHg}$ [11-14] definiert ist, sollte auch im Laufe der Zeit erhalten bleiben, um die Häufigkeit des Anlegens von Verbänden und die damit verbundene wirtschaftliche Belastung und den Arbeitsaufwand zu begrenzen. Bei der Auswahl des am besten für eine behandelte Person geeigneten Systems müssen jedoch verschiedene Parameter berücksichtigt werden. Insbesondere sollte ein ideales System das medizinische Personal in die Lage versetzen, die wirksamen und sicheren Druckverbände einfach, sicher und konsequent bei allen Behandelten anzuwenden, und die Akzeptanz durch diese sollte so hoch wie möglich sein, um ihre Mithilfe zu unterstützen.

In der Praxis erhält ein erheblicher Anteil der Behandelten immer noch keine angemessene oder überhaupt keine Kompressionstherapie [15-19]. Diesen Berichten zufolge sind die am häufigsten genannten Gründe für dieses Versagen der Behandlung die mangelnde Akzeptanz der Behandlung durch die erkrankte Person [15-19], eine zu komplizierte Anlegetechnik für einige Systeme oder Schwierigkeiten, bei anderen Systemen den richtigen Druck zu erreichen [16-18]. In diesem Zusammenhang wird nach wie vor aktiv nach neuen Lösungen gesucht, die den Komfort und die Akzeptanz der Behandelten verbessern oder das Anlegen von Verbänden erleichtern würden, ohne dass die anerkannte Wirksamkeit von Mehrkomponenten-Kompressionssystemen beeinträchtigt wird.

Als Vorstufe zur klinischen Entwicklung eines solchen Systems bestand das Ziel dieser Studie darin, bei gesunden Testpersonen die Leistungen einer neuen Generation von MehrkomponentenKompressionssystem, das in einem einzigen Verband geliefert wird, in Bezug auf Steifigkeit, Schnittstellendruck und Akzeptanz mit denen eines gut etablierten Kontroll-Mehrkomponentensystems, das häufig bei der Behandlung von venösen Beinulzera und Ödemen venösen Ursprungs eingesetzt wird [20-26], zu vergleichen.

\section{Patienten und Methoden}

\section{Aufbau der klinischen Studie}

Bei der FUSION-Studie handelte es sich um eine monozentrische, randomisierte, kontrollierte klinische Studie, die im September 2019 an gesunden Freiwilligen in Frankreich durchgeführt wurde.

\section{Kriterien für die Aufnahme in die Studie}

Teilnahmeberechtigt waren gesunde Freiwillige, die sich bereit erklärten, die Kompressionssysteme während des dreitägigen Studienzeitraums an beiden Beinen zu tragen. Männer und Frauen im Alter zwischen 18 und 65 Jahren mit einem Body-Mass-Index unter $30 \mathrm{~kg} / \mathrm{m}^{2}$ konnten teilnehmen. Beide Beine mussten einen Umfang zwischen 18 und $25 \mathrm{~cm}$ haben, eine gesunde Haut ohne Anzeichen von Hauterkrankungen (Ekzeme, Schuppenflechte ...), bei der klinischen Untersuchung tastbare hintere und beide Schienbein- und Fußpulse, einen Knöchel-Arm-Index von mehr 

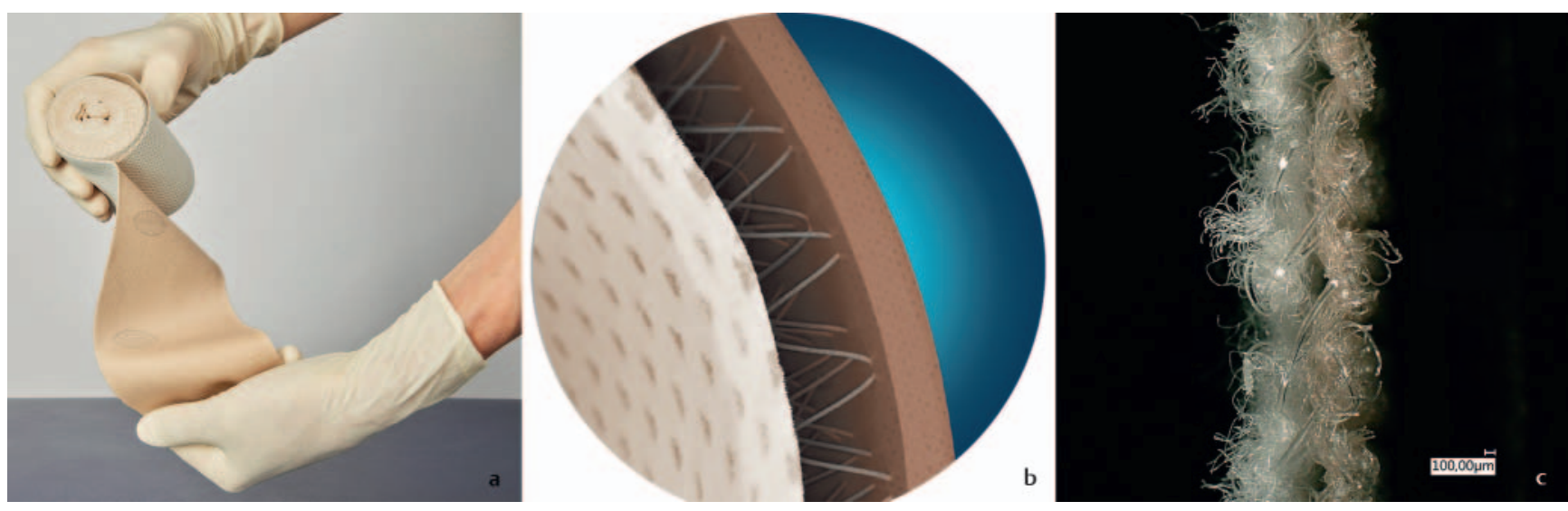

- Abb. 1 Präsentation des getesteten Systems. a Die beiden Seiten des neuen Dual Compression Systems, mit den aufgedruckten Druckindikatoren auf der beigen Außenseite. Quelle: Laboratories Urgo. b Schnittdarstellung der exklusiven Struktur des Mehrkomponentensystems, die zu einer einzigen Bandage führt. Quelle: Laboratories Urgo. c Mikroskopische Aufnahme eines Querschnitts des Systems (×50). Der Skalenbalken entspricht $100 \mu \mathrm{m}$. Quelle: Laboratories Urgo.

als 0,9 und weniger als 1,3 sowie einen venösen Echo-Doppler ohne erkennbare Anomalien. Personen wurden ausgeschlossen, wenn sie ein chronisches Insuffizienzstadium größer oder gleich 2 aufwiesen (CEAP-Klassifikation chronischer Venenerkrankungen), allergisch auf eine Komponente der zu untersuchenden Kompressionssysteme reagierten, an Diabetes oder einer fortschreitenden neoplastischen Erkrankung litten, eine arterielle Erkrankung, Krankheit oder Operation in der Anamnese nannten, die ihre Mobilität beeinträchtigt, auch wenn sie mit Medikamenten behandelt wurden, die ihre arterielle Durchblutung beeinträchtigen, oder eine systemische Behandlung hatten, die zum Auftreten von Ödemen der unteren Gliedmaßen führen kann, oder wenn sie in den letzten 15 Tagen einen Flug von mehr als sieben Stunden Dauer angetreten hatten. Schwangere oder stillende Frauen oder Frauen im gebärfähigen Alter, die nicht durch eine wirksame Verhütungsmethode geschützt waren, sowie Personen, die an einer anderen therapeutischen Studie teilgenommen haben, wurden ebenfalls ausgeschlossen.

\section{Untersuchte Kompressionssysteme}

Das in dieser Studie untersuchte neue Kompressionssystem (UrgoK1, Laboratoires Urgo, Frankreich) stellt eine neue Generation dualer Kompressionssysteme dar. Es soll angeblich einen dauerhaft hohen Arbeitsdruck und einen moderaten Ruhedruck bieten, der durch die Anwendung einer einzigen Bandage (mit zwei verschiedenen Seiten), die auf einer innovativen Technologie beruht, erreicht wird. Dieses Mehrkomponenten-Kompressionssystem besteht aus Polyamid-, Elasthan- und Polyestergarnen, die in einer exklusiven Struktur unter Verwendung einer dreidimensionalen Stricktechnologie miteinander verbunden sind ( $\bullet$ Abb.1). Um die korrekte Dehnung und Überlappung des Kompressionssystems zu steuern, weist der Verband auf seiner Außenseite einen optischen Indikator auf, der als PresSure-System bekannt ist (eine aufgedruckte Ellipse, die sich zu einem Kreis ausdehnt, wenn der richtige Druck ausgeübt wird, und die auch auf den anderen von der Firma vertriebenen Kompressionsverbänden zu sehen ist). Die Bandage ist mit einem Greifsystem ausgestattet, das dazu dient, sie vollständig anzulegen und das Kompressionssystem über einen längeren Zeitraum aufrechtzuerhalten.

Bei dem als Kontrolle verwendeten Kompressionssystem handelte es sich um ein Mehrkomponenten-Kompressionssystem, das eine gepolsterte Kurzzugbinde und eine kohäsive Langzugbinde kombiniert (UrgoK2, Laboratoires Urgo, Frankreich). Dieses System ist in der Behandlung von venösen Beinulzera und Ödemen venösen Ursprungs weit verbreitet (seit 2007 auf dem Markt) und sein Wirksamkeits- und Sicherheitsprofil wurde zuvor durch eine kontrollierte Zufallsuntersuchung (RCT) und mehrere Interventions- und Beobachtungsstudien nachgewiesen [20-28].

Alle angelegten Binden waren $10 \mathrm{~cm}$ breit und für Knöchel mit einem Umfang von 18 bis $25 \mathrm{~cm}$ geeignet.

\section{Randomisierung}

Ein Kompressionssystem wurde nach dem Zufallsprinzip an einer der unteren Gliedmaßen der Versuchspersonen angebracht, das andere Kompressionssystem an der anderen Gliedmaße. Die Randomisierungsliste wurde mittels eines computergenerierten Blockrandomisierung-Verfahrens von einem unabhängigen Unternehmen erstellt, das auch mit der Datenanalyse für diese Studie betraut war (Soladis, Lyon, Frankreich).

\section{Studienverlauf}

Alle Studienvisiten wurden in einem Untersuchungszentrum (Intertek, Paris, Frankreich) durchgeführt, das auf klinische Studien mit gesunden Freiwilligen spezialisiert ist. Die Teilnehmenden wurden von dieser akkreditierten Forschungseinrichtung vor der Aufnahme über dessen Website für klinische Studien und die Datenbank für gesunde Testpersonen rekrutiert.

Beim ersten Besuch wurden beide Kompressionssysteme von derselben erfahrenen Krankenschwester mit einer 50-prozentigen Überlappung gemäß den Herstelleranweisungen angelegt. Der erreichte Schnittstellendruck wurde jeweils unmittelbar nach der Anwendung überprüft. 
Der Schnittstellendruck zwischen den Kompressionssystemen und der Haut wurden am anatomischen Punkt B1 mit dem Picopress-Gerät (Microlabltalia, Italien) mit einem runden Drucksensor von $5 \mathrm{~cm}$ Durchmesser gemessen. Der anatomische Punkt B1 befindet sich auf der medialen Seite des Beins, hinter dem Schienbein, vor der Achillessehne, oberhalb des M. soleus und am Ursprung des M. gastrocnemius medialis. Der Sensor wurde während des gesamten Studienzeitraums an Ort und Stelle belassen und bei jeder Druckmessung an das Aufzeichnungsgerät angeschlossen.

Die Testpersonen wurden maximal drei Tage lang nachbeobachtet, einschließlich der Evaluierungsbesuche 4, 24, 48 und 72 Stunden nach Anlegen der Kompressionssysteme. Bei jeder Visite wurde der Schnittstellendruck sowohl in Rückenlage als auch im Stehen registriert und das Verrutschen der Binden gemessen, solange die Systeme noch angelegt waren. Das Komfortempfinden der Testpersonen wurde am Abend des Anwendungstages und am folgenden Tag sowie bei der letzten Visite erhoben. Bei der Abschlussbesichtigung wurden die Testpersonen auf der Grundlage der drei vorangegangenen Tage, an denen sie beide Systeme getragen hatten, gebeten, ihr bevorzugtes Kompressionssystem zu nennen.

\section{Ergebnisse}

Das primäre Ergebnis war der Anteil von Teilnehmenden mit einem SSI $\geq 10 \mathrm{mmHg}$, 48 Stunden nach der Anwendung des Kompressionssystems. Der SSI war definiert als die Differenz zwischen dem Arbeits- und dem Ruhe-Interfacedruck $(\mathrm{mmHg})$, die in stehender bzw. liegender Position erreicht wurde [11].

Zu den sekundären Ergebnissen gehörten die Veränderungen des jeweiligen Schnittstellendrucks und des SSI, der Anteil der Teilnehmenden mit einem SSI $\geq 10 \mathrm{mmHg}$ und die Bandagenverschiebung (in cm, seit dem Anlegen bei T0) bei jeder Bewertungsvisite. Der Komfort der Testpersonen beim Tragen der Kompressionssysteme wurde anhand der folgenden Wahrnehmungen bewertet: Bequemlichkeit beim Tragen mit Schuhen, Beweglichkeit des Knöchels, Wärmeempfinden, Juckreiz und Schmerzen. Die Testpersonen wurden nach ihrer Präferenz für eines der beiden Systeme in Bezug auf Ästhetik, Tastempfindung und Tragekomfort mit Schuhen gefragt.

Die Verträglichkeit der einzelnen Kompressionssysteme wurde anhand des von der untersuchenden ärztlichen Fachkraft bei der Abschlussuntersuchung untersuchten Hautzustands und der Anzahl und Art der während des Studienzeitraums gemeldeten unerwünschten Ereignisse bewertet.

\section{Statistische Analyse}

Die Datenanalyse wurde von einem unabhängigen Unternehmen (Soladis, Lyon, Frankreich) in Übereinstimmung mit dem statistischen Analyseplan durchgeführt.

Die statistische Einheit war das Bein der Testperson. Ausgehend von der Hypothese, dass $93 \%$ der Beine in der Kontrollgruppe einen $\mathrm{SSI} \geq 10 \mathrm{mmHg}$ aufwiesen, einem erwarteten Verhältnis zwischen den Gruppen von 1,05 und einer Nichtunterlegenheitsmarge von 15 Prozentpunkten, wurde berechnet, dass 22 Testpersonen (entsprechend 22 rechten und 22 linken Beinen) erfor- derlich waren, um die Nichtunterlegenheit des getesteten Systems gegenüber der Kontrolle mit einer Aussagekraft von $85 \%$ und einem Alpha-Risiko von $5 \%$ (einseitige Situation) nachzuweisen. Unter der Annahme einer Abbruchquote von etwa $10 \%$ sollten 25 Probanden (50 Beine) in diese klinische Studie einbezogen werden. Die Nichtunterlegenheitsanalyse wurde für die Population nach Protokoll (PP) durchgeführt, und eine Überlegenheitsanalyse war für die Intention-to-Treat-Population (ITT) geplant, wobei beide Tests mit dem Farrington-Manning-Score-Test durchgeführt wurden. Sekundäre Ergebnisse wurden mit dem Chi-Quadrat-Test oder Fisher's Exact Test für kategorische Parameter bewertet, wobei p-Werte unter 0,05 als signifikant angesehen wurden. Die Präferenzen wurden mit einem 95\%-Konfidenzintervall nach der Clopper-Pearson-Methode beschrieben. Alle Analysen wurden mit der SAS-Software (v9.4; SAS Institute, USA) durchgeführt.

\section{Ethische Grundlagen}

Diese klinische Studie wurde in Übereinstimmung mit den ethischen Grundsätzen der Deklaration von Helsinki und den europäischen und französischen Vorschriften für Medizinprodukte und Forschung am Menschen (ISO 14 155, europäische Richtlinien 93/42/EWG und Jardé-Gesetz) durchgeführt. Das Komitee für den Schutz von Personen (C.P.P.) West VI von Brest und die Nationale Agentur für die Sicherheit von Arzneimitteln und Gesundheitsprodukten (ANSM) gaben im August 2019 eine befürwortende Stellungnahme ab und erteilten die Genehmigung. Diese Studie wurde bei ClinicalTrials.gov unter der Nummer NCT04 159844 registriert. Vor der Teilnahme an der Studie wurde von allen Testpersonen eine schriftliche Einverständniserklärung eingeholt.

\section{Ergebnisse}

\section{Merkmale der Testpersonen bei Studienbeginn}

An dieser Studie nahmen 20 Frauen und 5 Männer teil, deren Alter zwischen 25 und 65 Jahren und deren BMI zwischen 18,1 und $29,3 \mathrm{~kg} / \mathrm{m}^{2}$ lag ( $\triangleright$ Tab. 1 ). Bei Studienbeginn hatten alle Testpersonen einen Knöchelumfang zwischen 18 und $24 \mathrm{~cm}$ und gesunde Haut. Tibial- und Fußpulse waren an allen Beinen tastbar und die durchgeführten Echo-Doppleruntersuchungen waren normal. Der mittlere ABPI-Wert betrug 1,1 $\pm 0,1$ in jeder Beingruppe.

Der bei der Anwendung erzielte jeweilige Schnittstellendruck war bei beiden Systemen vom ersten Versuch an angemessen und bestätigte eine einfache, konsistente und sichere Anwendung für jedes System. Die Mittelwerte waren zwischen den beiden Gruppen sowohl in der Arbeits- als auch in der Ruhestellung ähnlich, was die Vergleichbarkeit der Gruppen bei der Baseline bestätigt.

Während des Studienzeitraums wurde das Kontroll-Kompressionssystem bei einem Probanden nach 48 Stunden wegen mäßiger Schmerzen entfernt und zwei Druckmessungen nach 72 Stunden waren in der untersuchten Gruppe wegen verrutschter Verbände nicht möglich. 
- Tab. 1 Merkmale der Testpersonen und Beine zu Beginn der Studie sowie der bei T0 angewendete Druck auf die Oberfläche.

\begin{tabular}{|l|l|l|}
\hline Komprimierungssysteme & $\begin{array}{l}\text { geprüftes } \\
\text { System }\end{array}$ & $\begin{array}{l}\text { Kontroll- } \\
\text { system }\end{array}$ \\
\hline Anzahl der Testpersonen & 25 & \\
\hline Männlich/weiblich. $\mathrm{n}(\%)$ & $5(20 \%) / 20(80 \%)$ \\
\hline Alter (Jahre), Mittelwert \pm SD & $43,1 \pm 11,1$ \\
\hline BMI (kg/m²), Mittelwert \pm SD & $23,4 \pm 3,2$ & \\
\hline ABPl, Mittelwert \pm SD & $1,1 \pm 0,1$ & $1,1 \pm 0,1$ \\
\hline $\begin{array}{l}\text { Knöchelumfang am B-Punkt (cm), } \\
\text { Mittelwert } \pm \text { SD }\end{array}$ & $21,3 \pm 1,5$ & $21,3 \pm 1,5$ \\
\hline $\begin{array}{l}\text { Wadenumfang am B1-Punkt (cm), } \\
\text { Mittelwert } \pm \text { SD }\end{array}$ & $29,3 \pm 2,5$ & $29,1 \pm 2,6$ \\
\hline $\begin{array}{l}\text { Ruhedruck der Grenzfläche } \\
\text { bei T0 (mmHg), Mittelwert } \pm \text { SD }\end{array}$ & $42,1 \pm 1,9$ & $44,2 \pm 2,3$ \\
\hline $\begin{array}{l}\text { Arbeitsgrenzflächendruck } \\
\text { bei T0 (mmHg), Mittelwert } \pm \text { SD }\end{array}$ & $66,5 \pm 8,2$ & $65,3 \pm 5,5$ \\
\hline
\end{tabular}

\section{SSI nach 48 Stunden (primärer Endpunkt)}

Nach 48 Stunden wurde mit 88 \% der getesteten Systeme und mit $76 \%$ der Kontrollsysteme ein SSI $\geq 10 \mathrm{mmHg}$ erreicht ( $>$ Tab. 2). Die Nichtunterlegenheits-Hypothese wurde bestätigt: Das neue Kompressionssystem ist dem Kontrollsystem in Bezug auf Steifigkeit nicht unterlegen $(p=0,016)$.

Der an der ITT-Population durchgeführte Überlegenheitstest ergab keinen signifikanten Unterschied zwischen den beiden Gruppen ( $p=0,135)$.

\section{Veränderungen des Schnittstellendrucks und der SSI im Laufe der Zeit}

Der jeweilige mittlere Schnittstellendruck und SSI, die mit den beiden Kompressionssystemen bei jeder Visite erzielt wurden, sind in $>$ Tab. 3 aufgeführt.

Die Ergebnisse zeigen, dass:

- sich im Laufe der Zeit der jeweilige Schnittstellendruck bei beiden Systemen in ähnlicher Weise veränderte. Wie bei Kompressionssystemen häufig der Fall, wurde in den ersten Stunden nach der Anwendung ein Druckabfall beobachtet. Danach schien sich jedes der beiden Systeme zu stabilisieren, wobei in den folgenden Tagen ein langsamer Druckabfall von nur geringer Intensität zu verzeichnen war.

- Der Arbeitsdruck über 30 mmHg wurden mit beiden Kompressionssystemen bis zur letzten Visite, d. h. 72 Stunden nach Anwendung des Systems, aufrechterhalten, wobei die Mittelwerte zwischen T4 h und T72 h bei dem getesteten System von $45,5 \mathrm{mmHg}$ bis $33,0 \mathrm{mmHg}$ und bei dem Kontrollsystem von $49,4 \mathrm{mmHg}$ bis $35,0 \mathrm{mmHg}$ reichten.

- Der in beiden Gruppen jeweils gemessene Ruhedruck blieb in einem sicheren und gut verträglichen Bereich, der für Kompressionssysteme, die auch nachts getragen werden können, erwartet wird.
- Tab. 2 Vergleich des Anteils der behandelten Personen mit einem $\mathrm{SSI} \geq 10 \mathrm{mmHg}$ nach $48 \mathrm{~h}$.

\begin{tabular}{|l|l|l|}
\hline Komprimierungssysteme & $\begin{array}{l}\text { geprüftes } \\
\text { System }\end{array}$ & $\begin{array}{l}\text { Kontroll- } \\
\text { system }\end{array}$ \\
\hline $\mathrm{SSI} \geq 10 \mathrm{mmHg}$ nach $48 \mathrm{~h}, \mathrm{n} / \mathrm{N}(\%)$ & $22 / 25(88 \%)$ & $19 / 25(76 \%)$ \\
\hline Odds Ratio $[90 \%$ Konfidenzintervall] & $1,16[0,92 ; 1,50]$ \\
\hline $\begin{array}{l}\text { Nichtunterlegenheitstest } \\
\text { (PP-Analyse) }\end{array}$ & $\mathrm{P}=0,016$ \\
\hline
\end{tabular}

- Die Veränderungen des SSI mit beiden Kompressionssystemen folgten ebenfalls den gleichen Kurven ( $\bullet$ Abb. 2), wobei es keinen signifikanten Unterschied im Anteil der Probanden mit einem SSI $\geq 10 \mathrm{mmHg}$ bei jeder Visite gab ( $\triangleright$ Abb. 3 ). Selbst nach dreitägigem Tragen gewährleisten $68 \%$ der angelegten Kompressionssysteme noch einen $\mathrm{SSI} \geq 10 \mathrm{mmHg}$ (72\% des getesteten Systems und $64 \%$ des Kontrollsystems).

\section{Verrutschen des Verbandes mit der Zeit}

Während des dreitägigen Untersuchungszeitraums war das Verrutschen des Verbandes von der oberen Linie der Erstanwendung in beiden Gruppen minimal. Nach 4 bzw. 72 Stunden Tragezeit lagen die Mittelwerte zwischen 0,4 $\pm 0,5$ und 1,0 $\pm 1,2 \mathrm{~cm}$ in der Testgruppe (mit einem Spitzenwert von 2,0 \pm 3,1 nach 48 Stunden) und zwischen $0,8 \pm 0,8$ und $4,9 \pm 3,3 \mathrm{~cm}$ in der Kontrollgruppe, was auf den guten Halt beider Systeme beim Tragen am Tag und in der Nacht hinweist ( $\triangleright$ Abb. 4). Wie bereits erwähnt, wurde die Analyse bei der Abschlussuntersuchung an 23 Beinen in der Testgruppe durchgeführt, da zwei Systeme zum Zeitpunkt der Messungen nicht mehr angelegt waren, und an 24 Beinen in der Kontrollgruppe, da die Studie an den anderen Beinen vorzeitig beendet wurde.

\section{Komfort beim Tragen der Kompressionssysteme und Präferenz}

Das Komfortempfinden der Probanden unter der Kompressionstherapie war mit dem getesteten System generell besser als mit dem Kontrollsystem. Insbesondere wurden mit dem getesteten System signifikant seltener Beschwerden hinsichtlich der Einschränkung der Knöchelbeweglichkeit $(p=0,022)$, des Schuhanziehens $(p=0,025)$ und des Wärmeempfindens $(p=0,024)$ berichtet $(\triangleright$ Abb. 5), während hinsichtlich des Juckreizes oder des Schmerzempfindens kein Unterschied zwischen den beiden Systemen festgestellt wurde.

Nach dreitägigem Tragen beider Systeme gab die Mehrheit der gesunden Freiwilligen dem getesteten System gegenüber dem Kontrollsystem bei jedem der vier betrachteten Kriterien eine deutliche Präferenz ( $\mathbf{A b b} \mathbf{6} \mathbf{6})$. Das getestete System wurde von $76 \%$ (95\% Cl 54,9\%; 90,6\%) der Testpersonen als bequemer, von $84 \%$ (95\% Cl 63,9\%; 95,5\%) als angenehmer bei der Berührung, von $88 \%$ (95\% Cl 68,8\%; 97,5\%) als ästhetischer und von $88 \%$ (95\% Cl 68,8\%; 97,5\%) als einfacher beim Tragen von Schuhen beurteilt als das Kontrollsystem. 
- Tab. 3 Schnittstellendruck in stehender und liegender Position und SSI während des gesamten Studienzeitraums

\begin{tabular}{|c|c|c|c|c|c|c|}
\hline \multirow{2}{*}{$\begin{array}{l}\text { Komprimierungssysteme } \\
\text { Grenzflächendruck und SSI } \\
\text { (mmHg), Mittelwert } \pm \text { SD }\end{array}$} & \multicolumn{3}{|c|}{ geprüftes System } & \multicolumn{3}{|l|}{ Kontrollsystem } \\
\hline & Arbeitsdruck & Ruhedruck & SSI & Arbeitsdruck & Ruhedruck & SSI \\
\hline Bei T0 & $\begin{array}{l}n=25 \\
66,5 \pm 8,2\end{array}$ & $\begin{array}{l}n=25 \\
42,1 \pm 1,9\end{array}$ & $\begin{array}{l}n=25 \\
24,4 \pm 7,9\end{array}$ & $\begin{array}{l}n=25 \\
65,3 \pm 5,5\end{array}$ & $\begin{array}{l}n=25 \\
44,2 \pm 2,3\end{array}$ & $\begin{array}{l}n=25 \\
21,1 \pm 5,2\end{array}$ \\
\hline Nach $4 \mathrm{~h}$ & $\begin{array}{l}n=25 \\
45,5 \pm 5,3\end{array}$ & $\begin{array}{l}n=25 \\
25,3 \pm 2,9\end{array}$ & $\begin{array}{l}n=25 \\
20,3 \pm 4,9\end{array}$ & $\begin{array}{l}n=25 \\
49,4 \pm 4,1\end{array}$ & $\begin{array}{l}n=25 \\
30,9 \pm 2,8\end{array}$ & $\begin{array}{l}n=25 \\
18,5 \pm 3,9\end{array}$ \\
\hline Nach $24 \mathrm{~h}$ & $\begin{array}{l}n=25 \\
38,7 \pm 6,6\end{array}$ & $\begin{array}{l}n=25 \\
20,4 \pm 3,7\end{array}$ & $\begin{array}{l}n=25 \\
18,2 \pm 5,0\end{array}$ & $\begin{array}{l}n=25 \\
42,3 \pm 6,4\end{array}$ & $\begin{array}{l}n=25 \\
26,3 \pm 4,0\end{array}$ & $\begin{array}{l}n=25 \\
16,0 \pm 4,9\end{array}$ \\
\hline Nach $48 \mathrm{~h}$ & $\begin{array}{l}n=25 \\
35,1 \pm 7,6\end{array}$ & $\begin{array}{l}n=25 \\
18,4 \pm 4,4\end{array}$ & $\begin{array}{l}n=25 \\
16,7 \pm 4,8\end{array}$ & $\begin{array}{l}n=25 \\
37,7 \pm 8,4\end{array}$ & $\begin{array}{l}n=25 \\
23,9 \pm 4,7\end{array}$ & $\begin{array}{l}n=25 \\
13,8 \pm 5,4\end{array}$ \\
\hline Nach 72 h & $\begin{array}{l}n=23 \\
33,0 \pm 7,7\end{array}$ & $\begin{array}{l}n=23 \\
17,2 \pm 2,9\end{array}$ & $\begin{array}{l}n=23 \\
15,9 \pm 5,6\end{array}$ & $\begin{array}{l}n=24 \\
35,0 \pm 9,7\end{array}$ & $\begin{array}{l}n=24 \\
22,1 \pm 5,7\end{array}$ & $\begin{array}{l}n=24 \\
13,0 \pm 5,8\end{array}$ \\
\hline
\end{tabular}

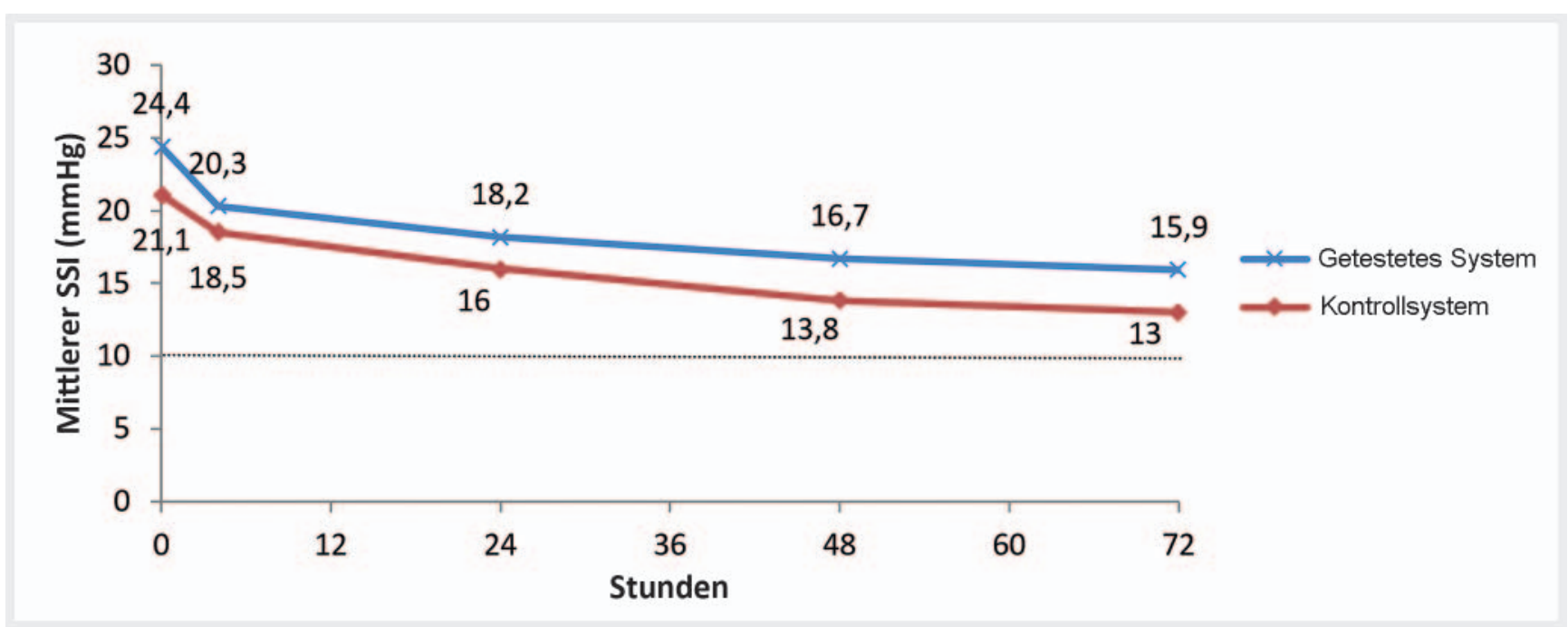

- Abb.2 Veränderungen des durchschnittlichen SSI während des Studienzeitraums.

\section{Sicherheitskriterien}

Beide Systeme waren sehr gut verträglich. Die klinische Untersuchung der Haut der Testpersonen während der Studie ergab keine Hauttrockenheit unter den Kompressionssystemen. Vier nicht schwerwiegende unerwünschte Ereignisse von geringer Intensität wurden vom Untersuchenden als dem Verfahren zuzuschreiben dokumentiert: das Auftreten einer Blase unter dem Drucksensor in der Testgruppe sowie das Auftreten einer Blase, eines Erythems und eines Ödems in der Kontrollgruppe. Ein am Ende der 48-stündigen Untersuchung gemeldeter mittelstarker Schmerz, der nach Einschätzung des Untersuchenden auf das Kompressionssystem zurückzuführen war, führte zum vorzeitigen Abbruch der Behandlung eines Beins in der Kontrollgruppe. Während des gesamten Studienzeitraums traten keine schwerwiegenden unerwünschten Ereignisse auf.

\section{Diskussion}

In der FUSION-Studie wurden erstmals die Leistungen einer neuen Generation von Mehrkomponenten-Kompressionssystemen, die zwei verschiedene Strukturen von Kompressionsschichten in einem einzigen Verband kombinieren, an gesunden Freiwilligen bewertet. Die Ergebnisse dieser RCT zeigten, dass dieses neue System ähnliche Eigenschaften aufweist wie das Kontrollsystem, ein Mehrkomponentensystem, das aus einer Kurzzugbinde und einer kohäsiven Binde besteht und in der Behandlung von venösen Beinulzera und Ödemen venösen Ursprungs weit verbreitet ist [21-23, 25]. Vier Stunden nach dem Anlegen wiesen beide Systeme eine ähnliche Steifigkeit auf, mit hohem Arbeitsdruck und mäßigem Ruhedruck. In den folgenden Tagen erreichten beide Systeme weiterhin einen SSI von über $10 \mathrm{mmHg}$ und hielten ihn aufrecht, was ihre starren Eigenschaften bestätigte. Die Akzeptanz des neuen Systems war jedoch deutlich höher als die des Kontrollsystems und die gesunden Testpersonen, die beide Syste- 


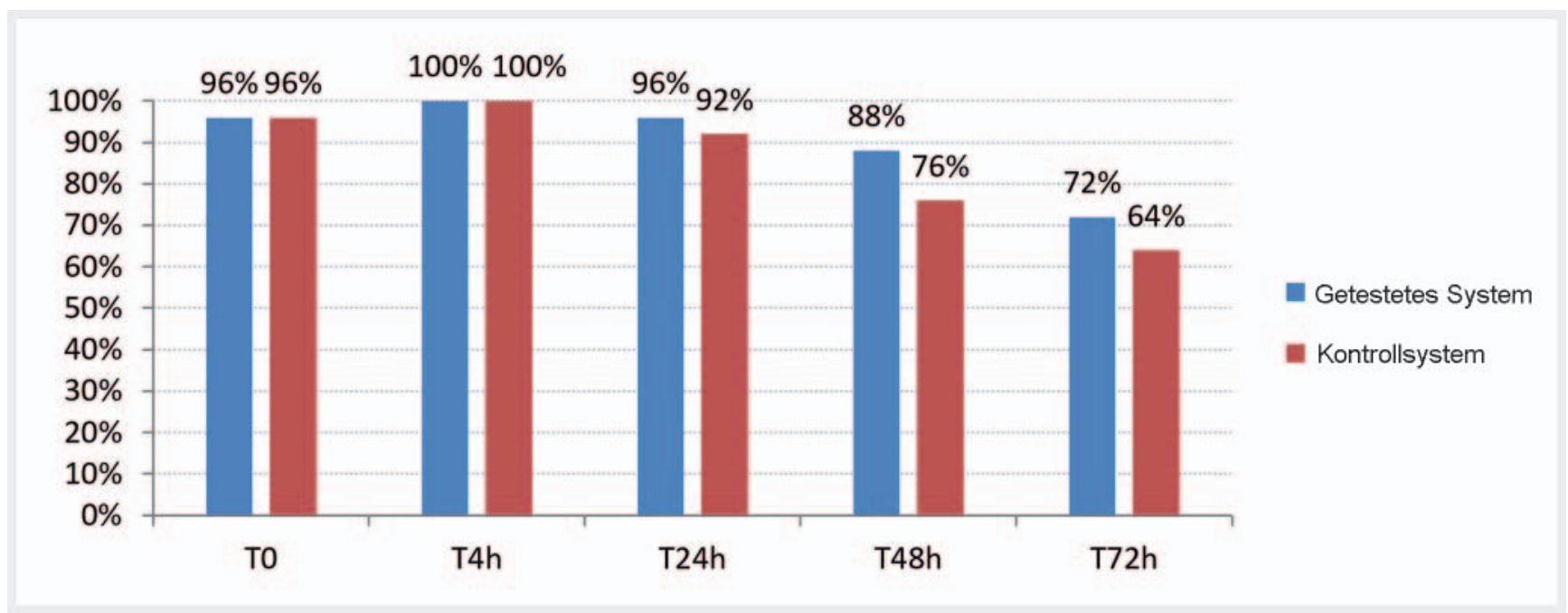

- Abb.3 Anteil der behandelten Personen mit einem SSI $\geq 10 \mathrm{mmHg}$ bei jeder Visite

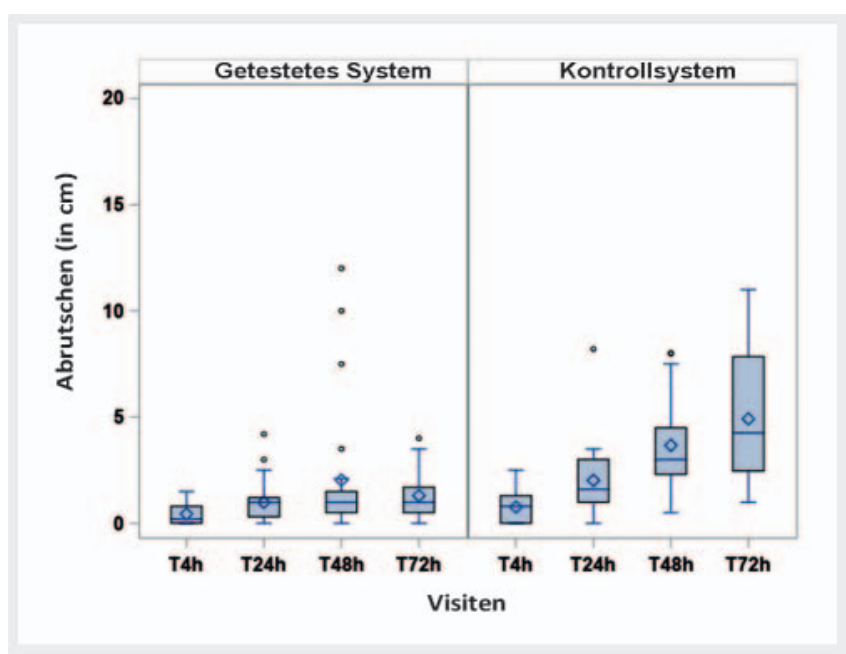

- Abb. 4 Verrutschen des Verbandes in cm bei jeder Visite. Die Rautensymbole geben die Mittelwerte an. Die Begrenzungen des Kastens zeigen die 25. und 75. Prozentile an, die Linie innerhalb des Kastens markiert den Median. Die Fehlerbalken zeigen die Extremwerte innerhalb des 1,5-fachen Interquartilsbereichs an. Die runden Symbole stellen Ausreißer dar, d. h. Werte, die über dem 1,5-Fachen des Interquartilsbereichs liegen.

me drei Tage und Nächte trugen, äußerten bei der abschließenden Bewertung eine deutliche Präferenz für das neue System.

Für Krankenhauspersonal sind die SSI und der angewandte Schnittstellendruck wichtige Indikatoren zur Einschätzung der klinischen Wirksamkeit, die von Kompressionssystemen erwartet werden kann [28-32]. Während die Prinzipien der Steifigkeit von Kompressionssystemen vor mehr als 15 Jahren eingeführt wurden und SSI schnell als Schlüsselelement bei der Charakterisierung von Kompressionssystemen anerkannt wurde [11-14, 33-35], gibt es nur wenige Studien, die die Veränderung von Arbeits- und Ruhedruck über mehrere Tage mit oder ohne den damit verbundenen SSI bewerten [28, 31, 36, 37]. In unserer Studie schienen sich der jeweils hohe Arbeitsdruck und der moderate Ruhedruck, die nach
4 Stunden erreicht wurden, in den folgenden Tagen zwischen beiden Mehrkomponentensystemen ähnlich zu verändern. Der vom getesteten System ausgeübte Druck an den Schnittstellen scheint in den ersten Stunden etwas stärker abzufallen als bei dem Kontrollsystem, aber dieser Unterschied war in Bezug auf die Hauptergebnisse nach 48 Stunden nicht signifikant. Der Verlust des Drucks an der Schnittstelle in den Stunden nach der Anwendung wurde bei allen Arten von Kompressionssystemen festgestellt [31, 36, 37]. Basierend auf diesen Studien wiesen Langzugbinden und elastische Systeme den geringsten Druckverlust über mehrere Tage auf, allerdings auch den geringsten Unterschied zwischen Arbeits- und Ruhedruck. Daher ist bekannt, dass sie während der Nacht nicht sehr gut stützen (aufgrund eines zu hohen Ruhedrucks in liegender Position) oder zu locker angelegt sind und tagsüber nicht immer genügend Arbeitsdruck ausüben (in stehender oder gehender Position) [36]. Außerdem verbessern sie aufgrund ihrer mangelnden Steifigkeit die Funktion der Venenpumpe nur wenig, da sie keinen Selbstmassageeffekt auf die Wade ausüben, wie von Mosti et al. bei Behandelten mit chronischer Veneninsuffizienz gezeigt wurde [38]. Im Gegensatz dazu zeichnen sich Kurzzugbinden durch eine hohe Steifigkeit, einen sehr starken Arbeitsdruck und einen sehr niedrigen Druck in Rückenlage aus, aber es wird auch berichtet, dass sie innerhalb der ersten Stunden des Tragens viel von ihrem Druck verlieren und daher häufiger neu angelegt werden müssen [31, 37]. Zwischen diesen Extremen werden Mehrkomponentenverbände in der Regel als guter Kompromiss angesehen, mit mäßigem Druckverlust im Laufe der Zeit, hohem Arbeitsdruck und mäßigem Druck, sodass sie Tag und Nacht getragen werden können [31]. Das in unserer Studie verwendete Kontrollsystem war zuvor von Protz et al. bewertet worden und war das einzige System unter anderen Mehrkomponentensystemen, die aus vier oder zwei Binden und Kurzzugbinden bestanden, das einen hohen Arbeitsdruck von über $40 \mathrm{mmHg}$ bis zu sieben Tagen aufrechterhalten konnte [31]. Ein Vergleich des von Kompressionssystemen aufgebrachten Drucks auf Grundlage der Ergebnisse verschiedener Studien ist aufgrund der Heterogenität der sie beeinflussenden Parameter 

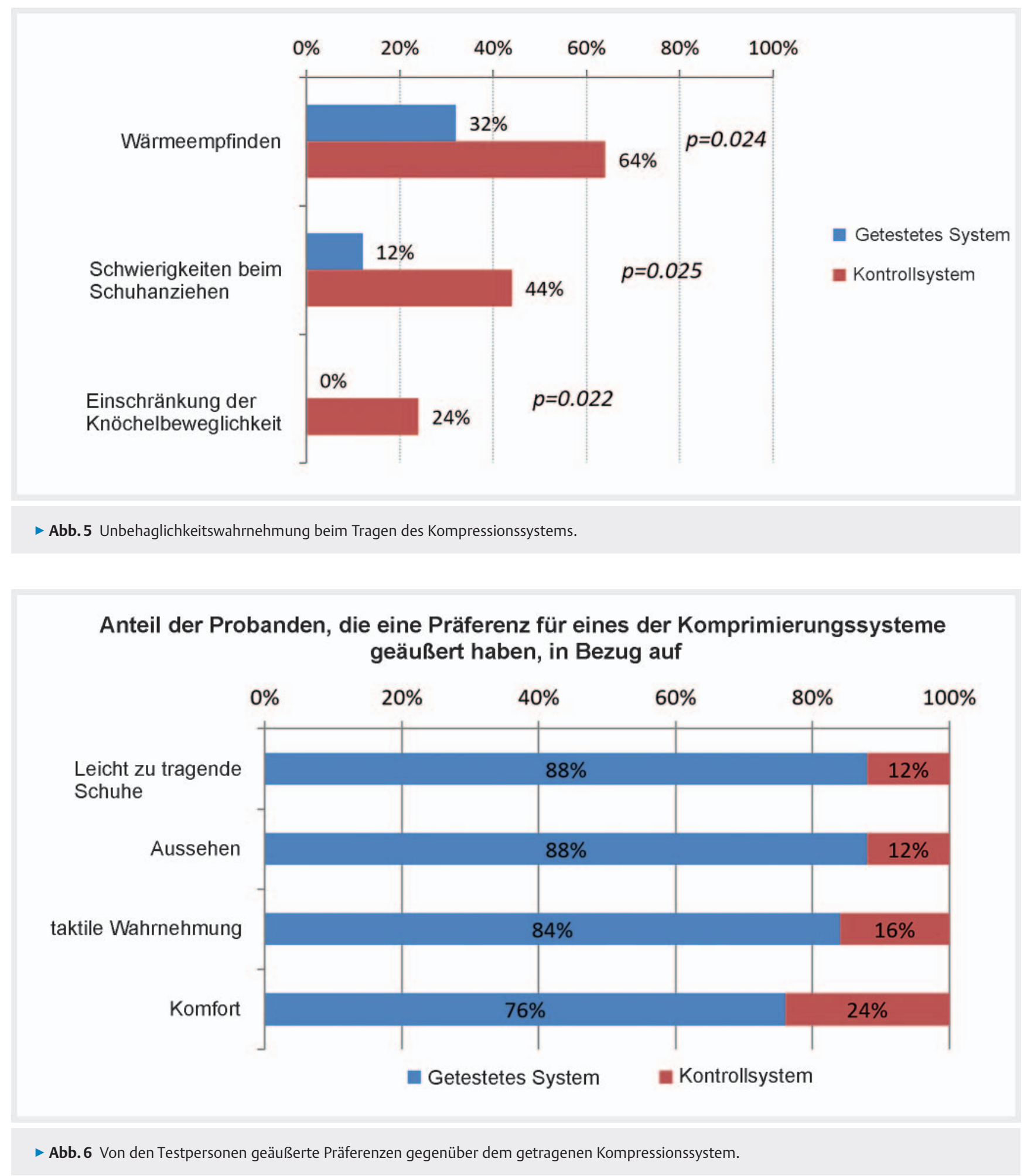

sehr schwierig [29, 32, 33, 39]. Dies gilt insbesondere für Systeme, bei denen die Gebrauchsanweisungen der Herstellfirmen einen erheblichen Spielraum in Bezug auf die Anlegetechniken, die Verwendung von Unterbinden, den Grad der Dehnung oder die Überlappung der Binden lassen. Mit nur einer erfahrenen Person, die für das Anlegen aller Binden zuständig war, einer gemeinsamen Anlegetechnik, der Verwendung des gleichen
Maßes an Überlappung und Dehnung für beide Systeme und der Anwendung der beiden Systeme an beiden Beinen jeder Testperson für den intraindividuellen Vergleich wurde diese RCT so konzipiert, dass die Anzahl der Variablen, die den Vergleich zwischen den beiden bewerteten Systemen einschränken könnten, minimiert wurde. 
Bei dem in dieser Studie verwendeten Kontrollsystem handelt es sich um ein gut etabliertes Mehrkomponentensystem. Sein Wirksamkeits- und Sicherheitsprofil wurde zuvor in einer RCT nachgewiesen, bei der im Vergleich zu einem Vierbindensystem ähnliche Wundverschlussraten erzielt wurden, während die Untersuchenden eine signifikant einfachere Anwendung des Zweibindensystems berichteten $(p=0,038)$ [20]. Seit dieser RCT wurden die gute Akzeptanz des Systems und seine Fähigkeit, einen hohen Arbeitsdruck und einen moderaten Ruhedruck bis zu sieben Tage lang sicher anzuwenden und aufrechtzuerhalten, in zahlreichen klinischen Studien und Beobachtungsstudien bestätigt [21-23, 25-28, 31]. Es hat sich gezeigt, dass visuelle Indikatoren, wie das PresSure-System, das auf den Binden der beiden in dieser Studie untersuchten Systeme abgebildet ist, das Anlegen von Binden mit dem Erreichen des jeweils vorgesehenen Drucks erheblich erleichtern und vereinfachen [40].

In unserer Studie wurden ähnlich gute Halteeigenschaften mit minimalem Verrutschen der Binden festgestellt, während beide Systeme Tag und Nacht getragen wurden. Wie in den meisten klinischen Studien zur Messung von Schnittstellendruck wurde auch diese Studie mit gesunden Testpersonen durchgeführt [28-31]. Das jüngere Alter und die größere Mobilität der Teilnehmenden im Vergleich zu Behandelten mit venösen Beinulzera können das Verrutschen der Binden verstärken und untermauern daher nur den hier berichteten Befund des guten Haltens beider Systeme auf Zeit. Da die Teilnehmer dieser Studie keine Ödeme aufwiesen, konnte die Notwendigkeit eines erneuten Anlegens der Systeme nach einer Ödemreduzierung (und damit einer Lockerung der angelegten Binden) nicht beurteilt werden. Die einfache Spiralmethode, mit der die bewerteten Verbände angelegt wurden, ist eine der am leichtesten zu beherrschenden Techniken. Bei schlecht kohäsiven Binden ist sie jedoch auch als eine Technik bekannt, die zu einem erheblichen Verrutschen des Verbandes und zu Druckverlusten führt, was erklärt, warum einige Binden immer noch eine komplexere und zeitaufwendigere Anlegetechnik, wie die Pütter-Technik, erfordern [29, 32, 39].

Das neue Mehrkomponenten-Kompressionssystem mit seiner einzigartigen Bandage erreicht ähnliche Leistungen wie das etablierte Mehrkomponentensystem, das zwei Bandagen miteinander verbindet. Ein Kompressionssystem in Form eines einzigen Verbandes wird sowohl von medizinischem Fachpersonal als auch von den Behandelten besonders geschätzt. Es bedeutet für beide einen geringeren Zeitaufwand für das Anlegen des Verbandes und damit auch weniger Zeit für die Mobilisierung zum Verbandswechsel. Wie in dieser Studie berichtet wurde, ging das Tragen eines einzigen Verbandes auch mit einem deutlich geringeren Wärmeempfinden, einer geringeren Einschränkung der Knöchelbeweglichkeit und einer Erleichterung des Schuhanziehens einher. Der von den Testpersonen empfundene verbesserte Komfort führte also dazu, dass die Mehrheit von ihnen das neue System deutlich bevorzugte. Da der Tragekomfort ein Schlüsselelement für die Einhaltung der Kompressionstherapie ist, könnte dieses neue Mehrkomponentensystem in einem Verband ein echter Vorteil bei der Behandlung von Kranken mit venösen Beinulzera und/ oder Ödemen venösen Ursprungs sein.

Die Ergebnisse dieser ersten klinischen Studie an gesunden Freiwilligen zeigen, dass dieses neue Kompressionssystem die wichtigsten Erwartungen an ein effizientes, gut verträgliches und gut akzeptiertes Kompressionssystem erfüllt. Die neue Generation des dualen Kompressionssystems zeigte während des 72-stündigen Studienzeitraums ähnliche Leistungen in Bezug auf Steifigkeit und Schnittstellendruck wie das Kontrollsystem, während beide Systeme Tag und Nacht getragen wurden. Beide Systeme wiesen ein gutes Sicherheitsprofil und gute Halteeigenschaften auf. Die Testpersonen gaben jedoch dem neuen System den Vorzug, das sie als bequemer und akzeptabler empfanden. Diese vielversprechenden Ergebnisse sprechen für die Leistungsfähigkeit des neuen Kompressionssystems, müssen aber noch in einer klinischen Studie an Kranken mit venösen Beinulzera und/oder Ödemen bestätigt werden.

\section{Finanzielle Unterstützung}

Diese Studie wurde durch einen Zuschuss von Urgo Research Innovation and Development unterstützt.

\section{Interessenkonflikt}

Olivier Tacca ist ein Mitarbeiter von Urgo Research Innovation and Development. Jean-Patrick Benigni war als Berater und Redner für pharmazeutische und andere Gesundheitsorganisationen tätig, unter anderem für Urgo Research Innovation and Development.

Datenmanagement und statistische Analysen wurden von Soladis, Lyon, Frankreich, unabhängig durchgeführt.

\section{Danksagung}

Wir danken Dr. Serge Bohbot, Director of Global Medical Affairs bei Laboratoires Urgo, für seine Unterstützung beim Studiendesign und der Durchsicht dieses Manuskripts sowie Laetitia Thomassin, PhD, und Nathalie Janot für ihre wertvollen Beiträge bei der Erstellung dieses Manuskripts.

\section{Literatur}

[1] Haute Autorité de Santé. [Medical compression in chronic venous conditions. Haute Autorité de Santé] [Article in French]. Ann Dermatol Venereol 2013; 140 (5): 393-396. doi:10.1016/j.annder.2013.03.004jkh

[2] Rabe E, Földi E, Gerlach H et al. Leitlinie: Medizinische Kompressionstherapie der Extremitäten mit Medizinischem Kompressionsstrumpf (MKS), Phlebologischem Kompressionsverband (PKV) und Medizinischen adaptiven Kompressionssystemen (MAK) AWMF-Registernummer: 037/005. https://www.awmf.org/uploads/tx_szleitlinien/037-005I_S3k_Medizini sche-Kompressionstherapie-MKS-PKV_2019-05.pdf (accessed March 15, 2021)

[3] Dissemond J, Assenheimer B, Bültemann A et al. Compression therapy in patients with venous leg ulcers. J Dtsch Dermatol Ges 2016; 14 (11): 1072-1087. doi:10.1111/ddg.13091

[4] Partsch B, Partsch H. Calf compression pressure required to achieve venous closure from supine to standing positions. J Vasc Surg 2005; 42 (4): 734-738. doi:10.1016/j.jvs.2005.06.030

[5] Partsch H, Stücker M, Vanscheidt W et al. Bedeutung des adäquaten Drucks in der Kompressionstherapie: Basis der erfolgreichen Behandlung [Importance of adequate pressure in compression therapy: Basis for successful treatment]. Hautarzt 2019; 70 (9): 707-714. doi:10.1007/ s00105-019-4413-9 
[6] O’Meara S, Cullum N, Nelson EA et al. Compression for venous leg ulcers. Cochrane Database Syst Rev 2012; 11: CD000265 doi:10.1002/ 14651858.CD000265.pub3

[7] O’Meara S, Tierney J, Cullum N et al. Four layer bandage compared with short stretch bandage for venous leg ulcers: Systematic review and meta-analysis of randomised controlled trials with data from individual patients. BMJ 2009; 338: b1344 doi:10.1136/bmj.b1344

[8] Haute Autorité de Santé. Prise en charge de l'ulcère de jambe à prédominance veineuse hors pansement. Recommendations. Juin 2006. https://www.has-sante.fr/upload/docs/application/pdf/ recommandations_finales_pdf.pdf (accessed November 30, 2020)

[9] O’Donnell TF Jr, Passman MA, Marston WA et al. Management of venous leg ulcers: clinical practice guidelines of the Society for Vascular Surgery (R) and the American Venous Forum. J Vasc Surg 2014; 60 (Suppl. 2): S3-S59. doi:10.1016/j.jvs.2014.04.049

[10] Scottish Intercollegiate Guidelines Network Management of chronic venous leg ulcers: A national clinical guideline. 2010 https://www.wwic. wales/uploads/files/documents/Professionals/Venous\%20Leg\%20Ulcer/ sign120.pdf (accessed November 30, 2020)

[11] Partsch $\mathrm{H}$. The static stiffness index: a simple method to assess the elastic property of compression material in vivo. Dermatol Surg 2005; 31 (6): 625-630. doi:10.1111/j.1524-4725.2005.31604

[12] Partsch H, Clark M, Bassez S et al. Measurement of lower leg compression in vivo: recommendations for the performance of measurements of interface pressure and stiffness: consensus statement. Dermatol Surg 2006; 32 (2): 224-233. doi:10.1111/j.1524-4725.2006.32039.x

[13] Partsch H, Schuren J, Mosti G et al. The Static Stiffness Index: an important parameter to characterise compression therapy in vivo. J Wound Care 2016; 25 (Suppl. 9): S4-S10. doi:10.12968/jowc.2016.25.Sup9.S4

[14] Chassagne F, Helouin-Desenne C, Molimard J et al. Superimposition of elastic and nonelastic compression bandages. J Vasc Surg Venous Lymphat Disord 2017; 5 (6): 851-858. doi:10.1016/j.jvsv.2017.07.006

[15] Renner R, Gebhardt C, Simon JC. Compliance hinsichtlich der Kompressionstherapie bei Patienten mit floridem Ulcus cruris venosum: Ergebnisse einer Querschnittsuntersuchung [Compliance to compression therapy in patients with existing venous leg ulcers. Results of a crosssectional study]. Med Klin (Munich) 2010; 105 (1): 1-6. doi:10.1007| s00063-010-1001-2

[16] Stansal A, Lazareth I, Michon Pasturel U et al. Compression therapy in 100 consecutive patients with venous leg ulcers. J Mal Vasc 2013; 38 (4): 252-258. doi:10.1016/j.jmv.2013.05.006

[17] Attal R, Mahé E, Bilan P et al. [Compression care for venous leg ulcers: Assessment of medical practices in 100 patients]. J Mal Vasc [French] 2015; 40 (3): 158-164. doi:10.1016/j.jmv.2015.03.006

[18] Le Gloanec H, Boye T, Placidi E. [Non-observance of medical compression in venous ulcers treatment]. Phlébologie [French] 2017; 70 (2): 3848 http://www.revue-phlebologie.org/donnees/portedocument/ mes_telechargements2.php?cparam $=905 \mathrm{~h} 6079 \mathrm{er} 6 \mathrm{~g} 3 \mathrm{o}$ i7yiy6br0u86sp7s4yf1jpw41a5tz6x91i6o0wfm06majii6p (accessed March 19, 2021)

[19] Pottier A, Le Thuaut A, Durand C et al. Evaluation of compression bandage application technique in patients with venous leg ulcers: A mixed methods observational study. Recherche en soins infirmiers 2019; 137 (2): 91-98. doi:10.3917/rsi.137.0091

[20] Lazareth I, Moffat C, Dissemond J et al. Efficacy of two compression systems in the management of VLUs: results of a European RCT. J Wound Care 2012; 21: 553-558. doi:10.12968/jowc.2012.21.11.553

[21] Lantis JC, Barrett C, Couch KS et al. A dual compression system: preliminary clinical insights from the US. J Wound Care 2020; 29 (Suppl. 9): S29-S37. doi:10.12968/jowc.2020.29.Sup9.S29

[22] Crebassa C, Allaert JF. [Venous leg ulcers treated in the community with a multicomponent compression system] [Article in French]. Phlebologie 2020; 73 (4): 67-77
[23] Stücker M, Münter KC, Erfurt-Berge C et al. Clinical evaluation of a multicomponent compression system: Results of a prospective, observational, multicentre study on 702 patients. J Wound Care 2021; in press

[24] Benigni JP, Lazareth I, Parpex P et al. Efficacy, safety, and acceptability of a new two-layer bandage system for venous leg ulcers. J Wound Care 2007; 16 (9): 385-390. doi:10.12968/jowc.2007.16.9.27866

[25] Conde-Montero E, Bohbot S, Grado Sanz R et al. Association of autologous punch grafting, TLC-NOSF dressing and multitype compression therapy to rapidly achieve wound closure in hard-to-heal venous leg ulcers. J Med Vasc 2020; 45 (6): 316-325. doi:10.1016/j.jdmv. 2020.10 .123

[26] Greenstein E, Tickner A. Addressing Compression Continuity, Consistency, and Comfort Using a Dual Compression System. Wound Manag Prev 2020; 66 (6): 4-7

[27] Hanna R, Bohbot S, Connolly N. A comparison of interface pressures of three compression bandage systems. Br ] Nurs 2008; 17 (20): S16-S24. doi:10.12968/bjon.2008.17.Sup9.31661

[28] Jünger M, Ladwig A, Bohbot S et al. Comparison of interface pressures of three compression bandaging systems used on healthy volunteers. J Wound Care 2009; 18 (11): 474-480. doi:10.12968/ jowc.2009.18.11.45000

[29] Lee AJ, Dale J], Ruckley CV et al. Compression therapy: effects of posture and application techniques on initial pressures delivered by bandages of different physical properties. Eur J Vasc Endovasc Surg 2006; 31 (5): 542-552. doi:10.1016/j.ejvs.2005.10.023

[30] Wong IK, Man MB, Chan OS et al. Andriessen A. Comparison of the interface pressure and stiffness of four types of compression systems. J Wound Care 2012; 21 (4): 161-167. doi:10.12968/ jowc.2012.21.4.161

[31] Protz K, Heyer K, Verheyen-Cronau I et al. Loss of interface pressure in various compression bandage systems over seven days. Dermatology 2014; 229 (4): 343-352. doi:10.1159/000365111

[32] Rimaud D, Convert R, Calmels P. In vivo measurement of compression bandage interface pressures: the first study. Ann Phys Rehabil Med 2014; 57 (6): 394-408. doi:10.1016/j.rehab.2014.06.005

[33] Mosti G, Mattaliano V, Partsch H. Influence of different materials in multicomponent bandages on pressure and stiffness of the final bandage. Dermatol Surg 2008; 34 (5): 631-639. doi:10.1111/ j.1524-4725.2007.34119.x

[34] Mosti GB, Mattaliano V. Simultaneous changes of leg circumference and interface pressure under different compression bandages. Eur J Vasc Endovasc Surg 2007; 33 (4): 476-482. doi:10.1016/j.ejvs.2006.11.035

[35] Hirai M, Koyama A, Miyazaki K et al. Interface pressure and stiffness in different combinations of compression material. Phlebology 2012; 27 (2): 82-89. doi:10.1258/phleb.2011.011010

[36] Hafner ], Botonakis I, Burg G. A comparison of multilayer bandage systems during rest, exercise, and over 2 days of wear time. Arch Dermatol 2000; 136 (7): 857-863. doi:10.1001/archderm.136.7.857

[37] Mosti G, Partsch H. Inelastic bandages maintain their hemodynamic effectiveness over time despite significant pressure loss. J Vasc Surg 2010; 52 (4): 925-931. doi:10.1016/j.jvs.2010.04.081

[38] Mosti G, Mattaliano V, Partsch H. Inelastic compression increases venous ejection fraction more than elastic bandages in patients with superficial venous reflux. Phlebology 2008; 23 (6): 287-294. doi:10.1258| phleb.2008.008009

[39] Miyazaki K, Hirai M, Koyama A et al. Interface pressure is affected by slippage of bandages at thigh. Int Angiol 2012; 31 (6): 544-549

[40] Sermsathanasawadi N, Tarapongpun T, Pianchareonsin R et al. Customizing elastic pressure bandages for reuse to a predetermined, sub-bandage pressure: A randomized controlled trial. Phlebology 2018; 33 (9): 627-635. doi:10.1177/0268355517746434 